\title{
Pruebas de cocción de pastas alimenticias elaboradas con harina de trigo - almidón de banano
}

\section{Cooking test of food pasta preapred with wheat fluor - banana starch -}

Edison Martínez Mora

Universidad Técnica de Machala (Machala - Ecuador)

Carmen Silverio Calderón Universidad de Guayaquil (Guayaquil - Ecuador)
Juliana Criollo Feijoo Universidad Técnica de Machala (Machala - Ecuador) juliana_cf2303@hotmail.com
Raúl Díaz Torres Universidad de Guayaquil (Guayaquil - Ecuador)

Revista Cumbres Vol.4 №1

Versión impresa ISSN 1390-9541

Versión electrónica ISSN 1390-3365

http://investigacion.utmachala.edu.ec/revistas/index.php/Cumbres 


\title{
RESUMEN
}

La investigación tuvo como objetivo, determinar el tiempo óptimo de cocción, pérdidas de peso y porcentaje de hinchamiento de pastas alimenticias cortas extruidas tipo fusilli, elaboradas con mezclas de harina de trigo durum (HT) y almidón nativo del cultivar de banano Cavendish (AB). Los tratamientos analizados tuvieron los siguientes porcentajes: 100 HT/ 0 AB (T0); 85 HT / 15 AB (T1); 70 HT / 30 AB (T2) y 55 HT / 45 AB (T3). El método de extracción del almidón fue por vía húmeda. La harina de trigo durum estuvo compuesta de $80 \%$ semolinas y $20 \%$ harinas de cola del trigo canadiense amber durum. La caracterización proximal se determinó por los métodos de la Association of Analytical Communities. Las pastas fueron elaboradas por extrusión, según la norma técnica ecuatoriana NTE INEN 1375, 2014. Las pruebas de cocción, se realizaron en agua a presión atmosférica, y la pérdida de peso por secado a $105{ }^{\circ} \mathrm{C}$ hasta peso constante. Los resultados obtenidos, demostraron que la incorporación de hasta un 30 \% de almidón por harina de trigo durum, las pastas no presentaron diferencia significativa $(p \geq 0,05)$ con el control o pasta tradicional.

Palabras clave: Almidón de banano, pastas alimenticias, pruebas de cocción

\begin{abstract}
The objective of this research was to determine the optimal cooking time, weight loss and swelling percentage in short-length fusilli type extruded pasta, elaborated from durum wheat flour (WF) and Cavendish banana native starch (BA) mixtures. The treatments analyzed consisted in the following percentages ratios: $100 \mathrm{WF} / 0 \mathrm{BA}$ (T0); $85 \mathrm{WF} / 15 \mathrm{BA}$ (T1); $70 \mathrm{WF} / 30 \mathrm{BA}$ (T2) and 55 WF / 45 BA (T3). Starch extraction technics used were the ones related to the so-called wet method. Durum wheat flour was composed of $80 \%$ semolines and 20\% Canadian wheat amber durum flour. Proximal characterization was determined following the Association of Analytical Communities protocols. Pasta was obtained by extrusion technologies, accordingly to the Ecuadorian technical standard NTE INEN 1375, 2014. Cooking tests were conducted in water at atmospheric pressure; weight loss tests were developed by drying at $105^{\circ} \mathrm{C}$ up to constant weighing. Results showed that in replacing up to $30 \%$ of the starch in a durum wheat flour matrix, Pasta featured no significant difference $(p \geq 0.05)$ when compared to the control treatment or traditional pasta.
\end{abstract}

Keywords: Banana starch, short-length pasta, cooking tests. 


\section{INTRODUCCIÓN}

Las pastas son un alimento tradicional en la dieta mediterránea, a base de cereales, generalmente recomendadas por su comodidad, palatabilidad y calidad nutricional y caracterizadas por un índice glucémico bajo que se atribuye a los cambios sucesivos en la estructura y a las interacciones entre los dos componentes principales: almidón y proteínas (Bustos, Pérez \& León, 2015). Las formas básicas de los productos de pasta como espagueti, lasagna, macarrones y otros, no se han alterado mucho durante siglos. Su preparación consiste en una masa cuyo ingrediente básico es la harina de trigo del granular durum que se une con agua, siendo optativa la adición de sal, huevos y otros ingredientes para la elaboración de la masa, la que posteriormente se conforma y se seca para obtener un producto que se cuece en agua hirviente antes de su consumo (Antognelli, 1980). Si bien cualquier material farináceo puede ser utilizado, la formulación occidental de este producto sigue la tradición heredada de Italia, esto es, el empleo de trigo extraduro (Triticum durum), indispensable para obtener el granular durum, que no es más que las sémolas mezcladas con las harinas de colas obtenidas en su proceso de molturación. Según la Norma Técnica Ecuatoriana NTE INEN 1375:2014, se entiende como pastas alimenticias o fideos a los productos no fermentados obtenidos por la mezcla de agua potable con harina de trigo durum y/o la inclusión de otros derivados amiláceos como materia prima sucedánea aptos para consumo humano, los cuales se someten a un proceso de prensado o de extrusión con un posterior secado o deshidratación del producto. La norma establece que el contenido máximo de humedad no deberá sobrepasar el 14 $\%$, el contenido de proteínas no superior a 10,5 \%, y como calidad microbiológica la ausencia de "E. coli", así como también, valores inferiores a 102 unidades formadoras de colonias de mohos y levaduras.

Las harinas compuestas son mezclas preparadas, principalmente con base de harina de trigo para la fabricación de productos panificados, de la repostería general y pastas alimenticias, que contienen todos los ingredientes y aditivos necesarios en la mezcla, que sirven para cumplir una determinada función técnica de elaboración de los mismos (Plasch y Bingen, 2008). El término harinas compuestas fue creado en 1964 por la Organización para la Agricultura y la Alimentación, cuando se reconoció la necesidad de buscar una solución para los países que no producen trigo (Elías, 1996), como es el caso del Ecuador. Estas harinas también, pueden prepararse a base de cereales y productos de origen vegetal distintos del trigo. Hay dos clases de harinas compuestas, la primera es una mezcla de harina de trigo con otras harinas diferente a esta (hasta en $40 \%$ ), pudiéndose agregar otros componentes. La segunda clase de harinas compuestas son aquellas que no contienen trigo y se preparan mezclando harinas obtenidas de tubérculos con harina de soya en menor nivel, u otras fuentes de proteína como suplemento. La sustitución parcial de harina de trigo en la elaboración de pastas alimenticias, ha sido objeto de numerosos estudios, por ejemplo, la incorporación de harina de arroz en la fabricación de fideos (Kahraman et al, 2008; Martí et a., 2010), el empleo de harina de guisantes (Wang et al, 1999), el uso combinado de 
harina de amaranto y almidón de maíz para producir pastas (Chillo y et al, 2007), el empleo como sustituto parcial de la sémola de trigo durum en la elaboración de pastas de harina de soya tostada (Baiano et al, 2011), empleo de una mezcla de harina de amaranto cruda : cocinada (Islas-Rubio, de la Barca, Cabrera-Chávez, Cota-Gastélum, \& Beta, 2014), uso de una mezcla de mijo, cebada y concentrado de proteína de suero (Yadav, Balasubramanian, Kaur, Anand, \& Singh, 2014), el empleo de harina de sorgo rojo (Khan, Yousif, Johnson, \& Gamlath, 2014) o harina de lentejas como sustituto parcial de harina de trigo (Hernández-Ordoñez, Hernández, \& Gelvez-Ordoñez, 2016). Sin embargo, no se encontraron estudios relacionados al uso de almidón de banano en la producción de pastas. El aprovechamiento de estas harinas, destinadas a la obtención de ingredientes con características nutricionales y funcionales, da un enfoque importante en el desarrollo de sistemas alimenticios hacia poblaciones con poca disponibilidad de niveles de energía y de nutrientes.

El objetivo de la investigación fue determinar el tiempo óptimo de cocción, las pérdidas de peso y el porcentaje de hinchamiento de pastas alimenticias cortas, elaboradas con mezclas de harina de trigo durum (HT) y almidón nativo del cultivar de banano Cavendish (AB).

\section{MATERIALES Y MÉTODOS}

\section{Ubicación del experimento y matera prima}

Las pastas alimenticias cortas del tipo "fusilli" se elaboraron en los laboratorios de alimentos de la Universidad Técnica de Ambato - Ecuador. Se utilizó como materia prima harina de trigo durum compuesta de $80 \%$ semolinas + $20 \%$ harinas de cola del trigo canadiense amber durum, fue adquirida en el mercado local. El almidón se obtuvo a partir de $200 \mathrm{~kg}$ del fruto de banano Cavendish.

\section{Aislamiento del almidón}

Para la obtención de almidón de banano Cavendish se utilizó el método de molienda húmeda, a escala piloto, se separó la cáscara de la pulpa y esta se cortó en rodajas de $1 \mathrm{~cm}$ de espesor, que fueron sumergidas en una solución con ácido ascórbico al 2 \% m/v. A continuación, se realizó la molienda, empleando una licuadora semi-industrial el producto resultante se cribó en mallas de calibre 0,$841 ; 0,149$ y 0,047 $\mathrm{mm}$ y el residuo obtenido en cada malla se lavó hasta que el líquido de lavado no presentó residuo aparente de almidón. La suspensión obtenida se separó por precipitación y luego de decantado, al almidón fue secado en un horno con ventilación forzada a temperatura entre 48 y $50{ }^{\circ} \mathrm{C}$ por 48 horas.

\section{Caracterización físico química del almidón nativo del cultivar de banano Cavendish}

La caracterización físico - química se hizo de acuerdo a los métodos oficiales 
descritos por la AOAC (1990): humedad (método 925.09), cenizas (método 923.03), proteínas (método 954.01), grasa cruda (método 920.39), carbohidratos totales por diferencia del $100 \%$ como extracto libre de nitrógeno

\section{Harina de trigo durum}

La harina de trigo durum compuesta de $80 \%$ semolinas $+20 \%$ harinas de cola del trigo canadiense amber durum, fue adquirida en el mercado local.

\section{Tratamientos experimentales}

Tabla 1. Mezclas harina de trigo durum/almidón de banano

\begin{tabular}{cc}
\hline Tratamiento & Mezclas \% \\
\hline T0 & $100 \mathrm{HT}^{\star}-0 \mathrm{AB}^{\star \star}$ \\
T1 & $85 \mathrm{HT}-15 \mathrm{AB}$ \\
$\mathrm{T} 2$ & $70 \mathrm{HT}-30 \mathrm{AB}$ \\
$\mathrm{T} 3$ & $55 \mathrm{HT}-45 \mathrm{AB}$ \\
\hline
\end{tabular}

Se elaboraron tres pastas en mezcla harina de trigo durum y almidón nativo de banano Cavendish, además una pasta control elaborada únicamente de harina de trigo durum (Tabla 1).

\section{Proceso de elaboración de pastas alimenticias}

Utilizando una extrusora marca Monferrina de 80 a $100 \mathrm{~kg}$ de capacidad. Las pastas se elaboraron según la Norma Técnica Ecuatoriana NTE INEN 1375, 2014. En los diferentes tratamientos, se mantuvo constante $30 \%$ de agua potable, no se utilizó huevo ni aditivos. El diagrama de proceso se muestra en la figura 1.

\section{Tiempo óptimo de cocción}

Se dispersaron $50 \mathrm{~g}$ de pasta alimenticia corta tipo fusilli muestra control o elaboradas con los diferentes porcentajes de mezclas harina de trigo - almidón nativo de banano las cuales se introdujeron por separado en $500 \mathrm{~mL}$ de agua en ebullición. Cada minuto, se tomaba un trozo de pasta entre dos vidrios de reloj y se comprimía. El tiempo óptimo de cocción (min) se estableció como aquel al cual desaparecía el color blanco después de la compresión (Granito, Pérez, \& Valero, 2014).

\section{Pérdida de peso por cocción}

Se calculó la cantidad de sólidos disueltos en el agua de cocción de las pastas alimenticias, para cada tratamiento de sustitución de la harina de trigo 
durum por el almidón nativo de banano. Para su determinación, el agua resultante de la cocción de las pastas, se decantó en un recipiente, que posteriormente se procedió a secar a $100{ }^{\circ} \mathrm{C}$ hasta peso constante (Granito et al, 2014).

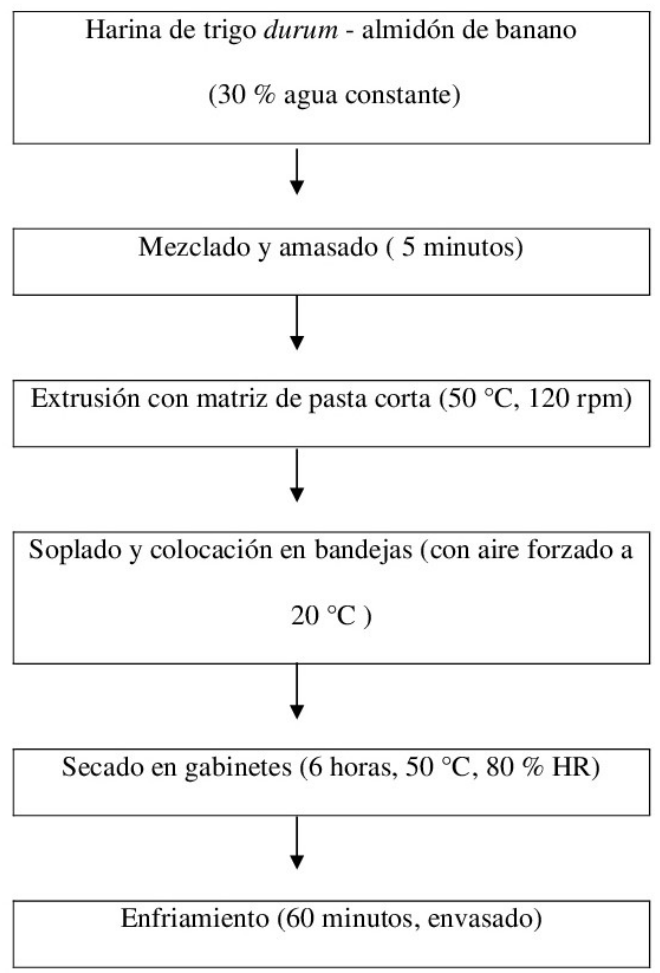

Figura 1. Diagrama de proceso de pastas cortas extruidas tipo "Fusilli"

\section{Porcentaje de hinchamiento}

Se empleó una modificación del método de (Granito et al, 2014). De cada uno de los tratamientos, se tomaron $50 \mathrm{~g}$ de pastas secas y se sometieron a cocción, utilizando el tiempo óptimo, se escurrió el líquido de cocción y se dejó enfriar hasta alcanzar la temperatura ambiente $\left(20^{\circ} \mathrm{C}\right)$. Seguidamente, se registró el peso de la pasta cocida (drenada) y pasta seca, utilizando una balanza analítica de la marca Mettler.

El porcentaje de hinchamiento de las pastas se comprobó mediante la siguiente ecuación:

$$
\% \text { de Hinchamiento }=\frac{\text { peso pasta cocida }- \text { peso de pasta seca }}{\text { peso de pasta seca }} \times 100 \text { [Ec.1] }
$$




\section{Análisis estadísticos}

El tratamiento estadístico de los datos se realizó por medio del análisis de varianza, así como una prueba de comparación de medias de Tukey, con un nivel de significancia $\mathrm{p} \leq$ 0,05 para establecer las diferencias entre las medias. Este análisis se realizó utilizando el paquete estadístico IBM SPSS ${ }^{\circledR} \mathrm{V}$ 19 (SPSS Institute Inc. Cary NC).

\section{RESULTADOS Y DISCUSIÓN}

\section{Composición química proximal del almidón nativo del cultivar de banano Cavendish}

En la tabla 2 se presentan los valores de composición proximal del almidón de banano Cavendish. Los valores obtenidos son similares a los reportados por García-Tejeda, Zamudio-Flores, Bello-Pérez, Romero-Bastida, \& Solorza-Feria, (2011), aunque el contenido de humedad del almidón nativo en este estudio es ligeramente mayor, lo que puede deberse a la diferencia en el método de secado. Respecto a las diferencias en el contenido de cenizas, grasa y proteína, estas pueden deberse tanto a la variedad como a las condiciones diferentes de cultivo.

\section{Pruebas de Cocción}

Tiempo óptimo de cocción

Los resultados mostrados en la tabla 3 permiten observar que el tiempo óptimo de cocción de las pastas alimenticias de los diferentes tratamientos, aumenta a medida que se incrementa la presencia del almidón nativo de banano en las pastas.

Tabla 2. Composición química proximal del almidón nativo del cultivar de banano Cavendish

\section{Componente (\%) Cavendish}

Humedad

Grasa

Proteína

Ceniza

Almidón Total
$9,32(0,9)$

$0,44(0,03)$

$0,86(0,07)$

$0,63(0,01)$

$88,75(0,22)$ 
La causa del aumento de temperatura, se debe a que el almidón nativo de banano posee elevadas temperaturas de gelatinización, por encima a la del almidón de arroz, maíz y trigo (Martínez, 2015), por lo que es de esperar que, a medida que este ingrediente se encuentra en mayor proporción en la mezcla, se necesite de mayor energía para su completa gelatinización, y por tanto, más tiempo de cocción. De Noni y Pagani (2010); reportaron que el tiempo óptimo de cocción para pastas alimenticias, aumenta a medida que se incorporan harinas compuestas a una masa. Del mismo modo, Sandoval (2011) reportó que los valores de tiempo óptimo de cocción para pastas cortas elaboradas únicamente con harina de trigo, está entre 10 y 12 minutos, y señaló además que, este tiempo es relativamente pequeño, debido a que la amilosa presente en sus almidones se encuentra alrededor de un $25 \%$, lo que beneficia a una rápida gelatinización, en este sentido, se comprobó que la incorporación de almidón nativo del cultivar de banano Cavendish, hasta el 30 \% no sobrepasa el límite de 12 minutos de cocción.

En el T3, la presencia de $45 \%$ de sustitución de la harina de trigo por almidón nativo de banano en la elaboración de pastas, contribuye al aumento del tiempo óptimo de cocción por encima de lo normal, y sería lógico esperar que, a mayores niveles de sustitución, el tiempo de cocción se prolongue. Otro factor que contribuye al aumento del tiempo de cocción, es el proceso de extrusión en la elaboración de pastas, ya que con ella se obtienen estructuras más compactas y menos porosas, que dificultan la transferencia de agua hacia el interior y, por consiguiente, alcanzar la gelatinización del nervio central de la pasta (Puppin et al, 2012). Uno de los atributos que más contribuyen a la alta demanda de las pastas alimenticias por los consumidores, es su pronta cocción, y por lo tanto, se le debe prestar mucha atención a este atributo al momento de diseñar y desarrollar una nueva pasta alimenticia.

\section{Pérdida de peso por cocción}

De acuerdo con los resultados que se muestran en la tabla 3, Las pérdidas de peso por cocción, estuvieron entre 8,13 \% (T0) y 13,11 \% (T3). La sustitución de harina de trigo hasta un 30 \% por almidón nativo de banano no produce cambios significativos en las pérdidas de peso por cocción. Estos resultados son similares a los reportados por Torres, Rodríguez, Guerra, \& Tabla 2. Composición química proximal del almidón nativo del cultivar de banano Cavendish

\begin{tabular}{cccc}
\hline \multirow{2}{*}{ Tratamiento } & Tiempo óptimo (min) & Pérdida de peso $(g)$ & Hinchamiento $(\%)$ \\
\hline T0 & $10,2^{\mathrm{a}}(0,5)$ & $8,13^{\mathrm{a}}(0,11)$ & $125,20^{\mathrm{a}}(8,20)$ \\
T1 & $10,1^{\mathrm{a}}(0,9)$ & $8,60^{\mathrm{a}}(0,10)$ & $130,09^{\mathrm{ab}}(5,90)$ \\
T2 & $11,8^{\mathrm{b}}(0,8)$ & $9,00^{\mathrm{a}}(0,98)$ & $141,79^{\mathrm{b}}(9,77)$ \\
T3 & $13,8^{\mathrm{b}}(0,1)$ & $13,11^{\mathrm{b}}(1,30)$ & $155,81^{\mathrm{c}}(13,30)$ \\
\hline
\end{tabular}

Los valores reportados son la media de 3 determinaciones. Los valores en paréntesis corresponden a las desviaciones estándar. Valores medios en la misma columna con distinta letra difieren significativamente $(\mathrm{p}<0,05)$. 
Granito, (2009) quienes reportaron que al aumentar la sustitución de harina de trigo, se incrementan las perdidas por cocción.

La sustitución parcial de la harina del granular durum, por otras fuentes sucedáneas, repercute en la calidad de las pastas alimenticias, siendo una de ellas, la pérdida de peso durante la cocción (Torres et al, 2007). Se ha señalado (Honesey, 1991) que estas pérdidas de peso no deben sobrepasar el $9 \%$, lo que en este trabajo se cumple hasta un $30 \%$ de sustitución.

\section{Porcentaje de hinchamiento de las pastas}

El porcentaje de hinchamiento de las pastas está relacionado con la capacidad de absorción de agua que posee el almidón. Durante la cocción, los gránulos de almidón absorben agua, lo que implica un aumento en su volumen.

En la tabla 3 se muestra la influencia de los diferentes tratamientos sobre los resultados del porcentaje de hinchamiento obtenido después de la cocción de las pastas alimenticias. El porcentaje de hinchamiento no presentó diferencia significativa con la muestra control con la incorporación de hasta un 30 \% (T2) de almidón.

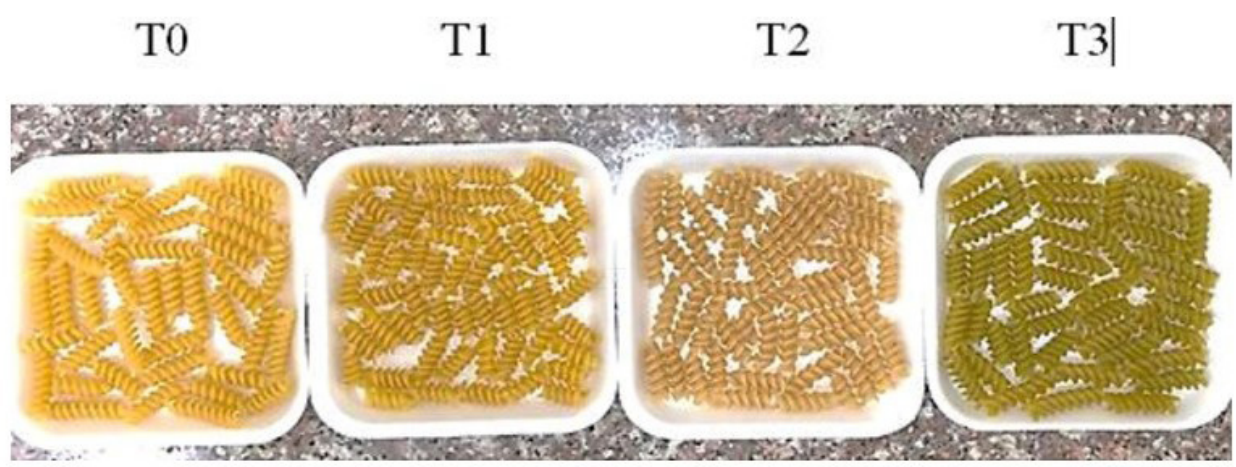

Figura 2. Imágenes de las pastas de cada tratamiento

\section{CONCLUSIONES}

Con la sustitución de hasta un 30 \% de harina de trigo por almidón de banano, el tiempo óptimo de cocción, las pérdidas de peso y el porcentaje de hinchamiento de pastas alimenticias cortas tipo fusilli extruidas, no presentan diferencia significativa $(\mathrm{p} \geq 0,05)$ con la pasta control o tradicional. Esta sustitución permite reducir los costos de producción, mediante la sustitución de una materia prima de importación, por una de producción nacional.

\section{REFERENCIAS BIBLIOGRÁFICAS}

AOAC. American Organization of Analytical Chemists. Official Methods of Analysis. 1990; 777 
Antognelli, C. (1980). The manufacture and applications of pasta as a food and as a food ingredient: a review. International Journal of Food Science \& Technology, 15(2), 125-145.

Baiano, A., Lamacchia, C., Fares, C., Terracone, C., \& La Notte, E. (2011). Cooking behaviour and acceptability of composite pasta made of semolina and toasted or partially defatted soy flour. LWT-Food Science and Technology, 44(4), 1226-1232.

Bustos, M. C., Perez, G. T., \& Leon, A. E. (2015). Structure and quality of pasta enriched with functional ingredients. Rsc Advances, 5(39), 30780-30792.

Chillo, S., Laverse, J., Falcone, P. M., \& Del Nobile, M. A. (2007). Effect of carboxymethylcellulose and pregelatinized corn starch on the quality of amaranthus spaghetti. Journal of Food Engineering, 83(4), 492-500.

De Noni, I., \& Pagani, M. A. (2010). Cooking properties and heat damage of dried pasta as influenced by raw material characteristics and processing conditions. Critical reviews in food science and nutrition, 50(5), 465-472.

Elías, L. G. (1996). Concepto y tecnologías para la elaboración y uso de harinas compuestas. Boletin de la Oficina Sanitaria Panamericana, 121(2), 179-182.

García-Tejeda, Y. V., Zamudio-Flores, P. B., Bello-Pérez, L. A., Romero-Bastida, C. A., \& Solorza-Feria, J. (2011). Oxidación del almidón nativo de plátano para su uso potencial en la fabricación de materiales de empaque biodegradables: caracterización física, química, térmica y morfológica. Revista Iberoamericana de Polímeros, 12(3), 125-135.

Granito, M., Pérez, S., \& Valero, Y. (2014). Calidad de cocción, aceptabilidad e índice glicémico de pasta larga enriquecida con leguminosas. Revista chilena de nutrición, 41(4), 425-432.

Hernández-Ordoñez, M., Hernández, C. A., \& Gelvez-Ordoñez, V. M. (2016). Macronutrientes de pasta alimenticia compuesta sustituyendo harina de trigo por harina de lentejas (Lens culinaris). Agronomía Colombiana, 34(1Supl), S1296-S1298.

Hoseney, R. C. (1991). Principios de Ciencia y Tecnología de los Cereales. Acribia. p.269-274.

Islas-Rubio, A. R., de la Barca, A. M. C., Cabrera-Chávez, F., Cota-Gastélum, A. G., \& Beta, T. (2014). Effect of semolina replacement with a raw: popped amaranth flour blend on cooking quality and texture of pasta. LWT-Food Science and Technology, 57(1), 217-222.

IBM SPSS ${ }^{\circledR}$ v 19 (SPSS Institute Inc. Cary NC) paquete estadístico

Kahraman, K., Sakıyan, O., Ozturk, S., Koksel, H., Sumnu, G., \& Dubat, A. (2008). Utilization of Mixolab ${ }^{\circledR}$ to predict the suitability of flours in terms of cake quality. European Food Research and Technology, 227(2), 565-570.

Khan, I., Yousif, A. M., Johnson, S. K., \& Gamlath, S. (2014). Effect of sorghum flour addition on in vitro starch digestibility, cooking quality, and consumer acceptability of durum wheat pasta. Journal of food science, 79(8), S1560-S1567.

Marti, A., Seetharaman, K., \& Pagani, M. A. (2010). Rice-based pasta: A comparison between conventional pasta-making and extrusion-cooking. Journal of Cereal Science, 52(3), 404-409.

Martínez, O., Lapo-Calderón, B., Pérez-Rodríguez, J., Zambrano-Cabrera, C., 
\& Maza-Valle, F. (2015). Mecanismo de gelatinización del almidón nativo de banano exportable del Ecuador. Revista Colombiana de Química, 44(2), $16-24$.

Norma Técnica Ecuatoriana, NTE INEN 1375:2014. (2014) Pastas Alimenticias o Fideos Secos. Requisitos.

Plasch, G. y Bingen, S. (2008). Mezclas y harinas preparadas. Soluciones prácticas: Disponible en: http://www.muehlenchemie.de/downloads-expertenwissen/mc-convenience-plasch-esp.pdf, Alemania.

Puppin, Z.R., Assuncao, B., Gandolfi, L., Selva, G., Martins, M. y Pratesi, R. (2012). Green banana pasta: An alternative for gluten-free diets. Journal of the Academy of Nutrition and Dietetic. 112(7), 1068-1072.

Sandoval, G. A. (2011). Desarrollo de mezclas farináceas de cereales (maíz, quinua y cebada) y papas ecuatorianas como sustitutos parciales del trigo importado para la elaboración de pan y fideos. Ambato: Universidad Técnica de Ambato. Facultad de Ciencia e Ingeniería en Alimentos.

Torres, A., Frías, J., Granito, M., \& Vidal-Valverde, C. (2007). Germinated Cajanus cajan seeds as ingredients in pasta products: Chemical, biological and sensory evaluation. Food Chemistry, 101(1), 202-211.

Torres, A., Rodríguez, M., Guerra, M., \& Granito, M. (2009). Factibilidad tecnológica de incorporar germen desgrasado de maíz en la elaboración de pasta corta. In Anales Venezolanos de Nutrición (Vol. 22, No. 1, pp. 25-31). Fundación Bengoa.

Wang, N., Bhirud, P. R., Sosulski, F. W., \& Tyler, R. T. (1999). Pasta-Like Product from Pea Flour by Twin-Screw Extrusion. Journal of Food Science, 64(4), 671-678.

Yadav, D. N., Balasubramanian, S., Kaur, J., Anand, T., \& Singh, A. K. (2014). Non-wheat pasta based on pearl millet flour containing barley and whey protein concentrate. Journal of Food Science and Technology, 51(10), 25922599.

7:3) 206-213.

Zamora, J. (2007). Antioxidantes: micronutrientes en lucha por la salud. Revista chilena de nutrición, 34(1), 17-26. 